\title{
Sentencia del Tribunal Constitucional recaída en recurso de inaplicabilidad del inciso tercero del artículo 23 y el inciso primero del artículo 24 del Decreto Ley No 3.063 sobre Rentas Municipales STC Rol No 2141-11, de 14 de marzo de 2013
}

\section{Doctrina}

- La generalidad de sujetos vinculados por la disposición legal no implica ausencia o falta de limitación del significado que el legislador quiso dar al pago de dicha patente municipal y a los sujetos obligados a ella. La regla general es que deben pagar patente municipal toda actividad lucrativa secundaria o terciaria.

- La propia jurisprudencia de esta Magistratura ha reconocido el principio de colaboración reglamentaria en materia impositiva, afirmando que "es la ley la encargada de precisar los elementos esenciales de la obligación tributaria, pudiendo la potestad reglamentaria de ejecución sólo desarrollar aspectos de detalle técnico que, por su propia naturaleza, el legislador no puede regular, pero que este debe delimitar con suficiente claridad y determinación.

- No es argumento convincente que la violación del principio de igualdad se produzca porque el legislador grave a una sociedad con el pago de patente municipal sólo "por su naturaleza". El legislador grava por igual a todas las sociedades que se encuentren en la misma condición que la requirente, sin discriminar o hacer diferencias de trato respecto de ellas.

"Profesor Titular de Derecho Constitucional, Facultad de Derecho, Universidad de Chile. 


\section{Requerimiento:}

El requerimiento de inaplicabilidad por inconstitucionalidad interpuesto impugna el Art. 23 inciso tercero y el Art. 24 inciso primero del Decreto Ley No 3.063 de 1979, Ley de Rentas Municipales. La primera de las normas establece que el Presidente de la República reglamentará la aplicación del Art. 23, el que establece la obligación de pagar patente municipal para quienes desarrollen actividades lucrativas secundarias y terciarias. Por su parte, la segunda de las normas impugnadas, seńala que se pagará la patente por un mismo contribuyente, con independencia de los giros que éste tenga y en caso de las sociedades de inversiones y de profesionales que no registren domicilio, se entenderá que éste es el registrado en el Servicio de Impuestos Internos.

A juicio del requerimiento, las normas impugnadas atentan en contra de las normas constitucionales de legalidad tributaria y la igualdad en la repartición de los tributos, ya que corresponde sólo al legislador crear tributos y establecer sus elementos esenciales de la misma, que en el caso se vulnerar al delegar en el Presidente de la República la aplicación de las normas en cuestión, ya que dicha delegación implica que sea el Presidente de la República el que configure finalmente la obligación tributaria en sus elementos esenciales.

Por otro lado, se señala que el artículo 24 establece la obligación del pago de patente a todas las sociedades de inversión y de profesionales, sin distinguir entre el tipo de actividad que ellas realizan, lo cual atenta en contra la igualdad en materia tributaria, puesto que la patente municipal es un impuesto que grava el desarrollo de una actividad, salvo en el caso de las sociedades referidas, caso en el que se aplicaría el tributo atendiendo al tipo de sociedad y no a la actividad que ellas realizan.

\section{Conflicto Constitucional:}

En cuanto a la fijación del conflicto, la sentencia hace un resumen de los requisitos de la acción de inaplicabilidad y la forma en cómo estos se cumplen en el caso de autos, especificando las normas legales impugnadas y las normas constitucionales supuestamente infringidas:

"SEGUNDO: Que, en relación al primer requisito, en el caso de autos se solicita la inaplicabilidad por inconstitucionalidad de los artículos 23, inciso tercero, y 24, inciso primero, del D.L. $N^{\circ} 3.063$, sobre Rentas Municipales, en la causa sobre reclamo de ilegalidad caratulada "Inversiones Luma Limitada con De la Maza Chadwick, Francisco", que se encuentra actualmente pendiente en recurso de casación en el fondo ante la Corte Suprema, bajo el Rol de Ingreso No 10.268-2011;

TERCERO: Que, en lo que se refiere al segundo requisito, la inaplicabilidad es formulada por la sociedad Inversiones Luma Limitada, reclamante de ilegalidad y recurrente de casación en el fondo en el asunto de fondo sometido a conocimiento de esta Magistratura; 
CUARTO: Que, en el caso de autos, se impugnan los artículos 23, inciso tercero, y 24, inciso primero, del D.L. $N^{\circ} 3.063$, preceptos que pueden resultar decisivos para la resolución del asunto y cuyos textos se transcribieron en la parte expositiva de esta sentencia;

QUINTO: Que la requirente sostiene que la aplicación de los preceptos legales aludidos vulnera las garantías de legalidad tributaria y de igualdad en la aplicación de los tributos;"

\section{Ratio Decidendi}

\section{Reserva Legal Tributaria:}

En cuanto a la vulneración de la reserva legal en materia tributaria, la sentencia señala que de ella se derivan dos situaciones, primero, la indeterminación de la norma legal y segundo, una delegación al Presidente de la República que sería inconstitucional:

"NOVENO: Que de esta impugnación se derivan dos cuestionamientos diferentes al principio de reserva legal tributaria. Primero, que el propio legislador realizó una insuficiente determinación del tributo. Segundo, que esta indeterminación es cerrada normativamente recurriendo al expediente de la remisión reglamentaria, cuestión impedida por el principio de reserva legal absoluta, principio que exige del legislador una regulación 'con la mayor amplitud, profundidad y precisión que resulte compatible con las caracteristicas de la ley como una categoría, diferenciada e inconfundible, de norma jurídica' (STC Rol $N^{\circ} 370$, considerando decimoséptimo);"

En cuanto a la primera de las aristas, es rechazado el reclamo por la sentencia, puesto que una cosa es que el sujeto destinatario de la norma, y obligado al pago del tributo, sea amplio y una cuestión diferente es que sea indeterminado. En el caso de autos, el sujeto obligado es amplio, ya que se señala que "toda profesión, oficio, industria...", pero no se haya indeterminado, puesto que queda absolutamente claro en la norma legal quienes deben pagar patente municipal:

"DÉCIMO: Que, en relación a la indeterminación con que habría procedido el legislador al fijar el tributo, la "amplitud" de sujetos obligados por una disposición legal no es sinónimo de existencia de una zona gris o no determinada de interpretación de una disposición que deba reglamentar otra potestad normativa;

UNDÉCIMO: Que lo anterior implica reconocer que hay algunos elementos esenciales que deben formar parte de la determinación del contenido fundamental del tributo. Ellos son la obligación tributaria, los sujetos de la obligación -tanto activo como pasivo-, el hecho gravado, el objeto de la obligación, la base imponible, la tasa y, en general, otros elementos especificos, dependiendo del tributo;

DUODÉCIMO: Que, a partir de los criterios enunciados por este propio Tribunal Constitucional los destinatarios de la obligación tributaria de pago de patente municipal los establece el decreto ley $N^{\circ} 3.063$, sin dejar márgenes inconstitucionales de indeterminación 
normativa. La generalidad de sujetos vinculados por la disposición legal no implica ausencia o falta de limitación del significado que el legislador quiso dar al pago de dicha patente municipal y a los sujetos obligados a ella. La regla general es que deben pagar patente municipal toda actividad lucrativa secundaria o terciaria;

DECIMOTERCERO: Que el inciso final del artículo 23, impugnado de inconstitucionalidad en su aplicación al caso de autos, no debe ser leido parcialmente. De la lectura de las disposiciones del decreto ley que contiene el inciso tercero del artículo 23, es claro y no deja lugar a dudas, ni apertura normativa a la potestad reglamentaria, en cuanto a quiénes son obligados al pago de patente municipal. Efectivamente, éste grava el ejercicio de "toda profesión, oficio, industria, comercio, arte o cualquier otra actividad lucrativa secundaria o terciaria, sea cual fuere su naturaleza o denominación" (articulo 23, inciso primero, y sin perjuicio de las actividades primarias gravadas en las circunstancias descritas en el inciso segundo), dentro de las cualesel propio legislador incluye a las 'sociedades de inversión' (artículo 24, inciso primero), dejando exentas a "las personas jurídicas que realicen acciones de beneficencia, de culto religioso, culturales, de ayuda mutua de sus asociados, artísticas o deportivas no profesionales y de promoción de intereses comunitarios."

En cuanto a la presunta vulneración de la reserva legal absoluta en materia tributaria, la sentencia igualmente rechaza este capítulo de impugnación, ya que considera que la delegación no ha sobrepasado el desarrollo legislativo correspondiente. Esto, por cuanto la norma impugnada señala que es competencia del Presidente regular solamente la "aplicación" de la misma, y la potestad reglamentaria de ejecución la tiene el Presidente incluso sin la necesidad de que se la otorguen normas especiales, con lo cual la norma impugnada no hace sino especificar una competencia que el Presidente ya tiene, sin que ello viole la reserva legal en materia tributaria:

"DECIMOSÉPTIMO: Que en lo relativo a la impugnación por presunta violación del principio de reserva legal absoluta en materia tributaria, cabe replicar las siguientes consideraciones basadas en la jurisprudencia de este Tribunal;

DECIMOCTAVO: Que el test esencial a verificar es si el desarrollo normativo del legislador ha sido sobrepasado en la colaboración reglamentaria. Por tanto, hay que verificar si concurre en este caso la regla de determinación y especificidad de la obligación tributaria (STC Rol $N^{\circ} 465$, considerando $25^{\circ}$ ). El argumento que concluye con la determinación de un tributo es que este sea especifico en la afectación de los derechos fundamentales involucrados y que aquello se realice por ley adecuadamente ejecutada por normas reglamentarias;

DECIMONOVENO: Que, en primer lugar, el artículo 23, inciso tercero, del D.L $N^{\circ}$ 3.063 sostiene que "el Presidente de la República reglamentará la aplicación de este artículo". Ello no es más que la aplicación de la potestad reglamentaria de ejecución de la ley que todo cuerpo legal envuelve;

VIGESIMO: Que, en efecto, conforme a lo dispuesto en el articulo $32 N^{\circ} 6$ de la Carta Fundamental, son 'atribuciones especiales del Presidente de la República: Ejercer la potestad reglamentaria en todas aquellas materias que no sean propias del dominio legal, sin 
perjuicio de la facultad de dictar los demás reglamentos, decretos e instrucciones que crea convenientes para la ejecución de las leyes'.

VIGESIMOPRIMERO: Que la potestad reglamentaria de ejecución existe, incluso, sin necesidad de reconocimiento legal (en sentido estricto) expreso, porque la propia disposición constitucional la estatuye a objeto de darle plena eficacia a los mandatos del legislador;

VIGESIMOSEGUNDO: Que la propia jurisprudencia de esta Magistratura ha reconocido el principio de colaboración reglamentaria en materia impositiva, afirmando que "es la ley la encargada de precisar los elementos esenciales de la obligación tributaria, pudiendo la potestad reglamentaria de ejecución sólo desarrollar aspectos de detalle técnico que, por su propia naturaleza, el legislador no puede regular, pero que este debe delimitar con suficiente claridad y determinación" (STC Rol No 759, considerando 25);"

\section{Igualdad ante la Ley:}

En cuanto al reproche de violación a la igualdad ante la ley en materia tributaria, la sentencia parte señalando que primeramente debe dilucidarse si es que la norma legal establece una diferencia y luego, en segundo lugar, si es que la misma resulta ser arbitraria:

"VIGESIMOCTAVO: Que es doctrina asentada de este Tribunal que el principio de igualdad consiste en 'que las normas jurídicas deben ser iguales para todas las personas que se encuentren en las mismas circunstancias y, consecuencialmente, diversas para aquellas que se encuentren en situaciones diferentes. No se trata, por consiguiente, de una igualdad absoluta sino que ha de aplicarse la ley en cada caso conforme a las diferencias constitutivas del mismo. La igualdad supone, por lo tanto, la distinción razonable entre quienes no se encuentren en la misma condición' (STC Rol $N^{\circ} 1254$, considerando 46 $)$;

VIGESIMONOVENO: Que no es argumento convincente que la violación del principio de igualdad se produzca porque el legislador grave a una sociedad con el pago de patente municipal sólo "por su naturaleza". El legislador grava por igual a todas las sociedades que se encuentren en la misma condición que la requirente, sin discriminar o hacer diferencias de trato respecto de ellas;

TRIGÉSIMO: Que esta Magistratura ha fallado que para efectos "de dilucidar si, en el conflicto que se ha planteado, se produce una infracción al derecho a la igualdad ante la ley, es necesario determinar, en primer lugar, si realmente estamos frente a una discriminación o diferencia de trato entre personas que se encuentran en una situación similar para, luego, examinar si tal diferencia tiene el carácter de arbitraria importando una transgresión a la Carta Fundamental" (STC Rol No 1340 y en el mismo sentido, entre otras, STC roles $N^{\mathrm{o}}$ 790, 825, 829);"

La sentencia concluye luego que la norma impugnada no crea una discriminación arbitraria, puesto que somete a todas las sociedades que se encuentran en la misma situación a las mismas normas, y que en cualquier caso, no se establece un tributo, sino que solamente normas procedimentales para 
su operación, las que, como se ha dicho, son iguales para todas las sociedades que estén en el supuesto de hecho de la norma.

"TRIGESIMOPRIMERO: Que en la presente causa siquiera existe diferencia de trato respecto de la sociedad requirente con otras que se encuentren en condición similar. Como ya se afirmó, toda sociedad que se encuentre en la misma posición jurídica que la requirente estará obligada, en principio, al pago de patente municipal, y corresponderá a los tribunales ordinarios determinarlo;

TRIGESIMOSEGUNDO: Que, por lo demás, el artículo 24, inciso primero, del D.L $N^{\circ}$ 3.063 no grava especialmente a las sociedades de inversión, sino que establece una norma procedimental sobre el domicilio del pago de patente municipal;

TRIGESIMOTERCERO: Que la historia de la Ley 20.033, que modificó la disposición requerida, es clara en ese respecto, cuando afirma que la iniciativa "incorpora dos oraciones finales al inciso primero (que señala que la patente grava la actividad que se ejerce por un mismo contribuyente en su local, oficina o establecimiento), en el sentido de precisar que tratándose de las sociedades de inversiones o de profesionales que no registren domicilio comercial, la patente se pagará en la comuna registrada por el contribuyente ante el Servicio de Impuestos Internos, el que aportará esta información a los municipios en la forma que se señala, norma que fue aprobada por idéntico quórum.

Al margen de precisar que la disposición en comento se limita a perfeccionar el sistema de cobranza de la patente, sin constituir un nuevo impuesto, el representante del Ejecutivo expresó que hoy dia existen aproximadamente 150 mil sociedades de inversiones y de profesionales, gran parte de las cuales no tiene domicilio comercial, lo que dificulta o hace imposible el cobro en cuestión. Dada la considerable evasión tributaria en la materia, por este concepto se espera recaudar unos \$4.500 millones" (Primer Informe de la Comisión de Gobierno Interior en primer trámite constitucional, Boletín 2892-06) [Énfasis agregado];"

\section{Prevención de los Ministros Sres. Bertelsen Repetto, Venegas Palacios y Aróstica Maldonado}

Los Ministros Raúl Bertelsen, Marcelo Venegas e Iván Aróstica, llaman la atención en que el Decreto Supremo dictado en el ańo 2009, para reemplazar el reglamento de 1981, fue devuelto por la Contraloría General de la República, estando dentro de sus objeciones las normas relativas a las sociedades de inversión:

"Entre otras objeciones consideró el Organismo Contralor, respecto de una de las disposiciones del reglamento cuestionado, que "no resulta procedente la mención efectuada en dicho literal, a las 'sociedades de inversión' como sujetos de la referida patente, por cuanto los artículos 23 y 24 del decreto ley $N^{\circ} 3.063$, de 1979, gravan las actividades que indican independientemente de la naturaleza jurídica de los sujetos que las realicen".

Ante esto, el Presidente de la República debió haber recurrido al Tribunal Constitucional, cuestión que no hizo, ni tampoco fue ello requerido por las Municipalidades, por lo que los Ministros entienden 
que la representación de la Contraloría General de la República quedó a firme y la norma en cuestión jamás formó parte del ordenamiento jurídico vigente:

"De esta forma, se configuró la hipótesis contemplada en el inciso tercero del artículo 99 de la Carta Fundamental, merced al cual si la representación en grado de toma de razón tuviere lugar con respecto a un decreto por ser contrario a la Constitución, el Presidente de la República no tendrá la facultad de insistir, y en caso de no conformarse con esa objeción de la Contraloría General "deberá remitir los antecedentes al Tribunal Constitucional dentro del plazo de diez dias, a fin de que éste resuelva la controversia”.

Tal requerimiento no se produjo por el Jefe de Estado, único órgano legitimado para ocurrir en ese evento ante este Tribunal Constitucional, con arreglo a lo prescrito en el artículo 109 de la ley orgánica constitucional $N^{\circ}$ 17.997. Tampoco se sabe que las Municipalidades interesadas hayan impetrado al Presidente de la República ejercer dicha impugnación, en contra de aquel reparo efectuado por la Contraloría General en el citado oficio $N^{\circ} 25.825$.

Al no haber tenido lugar la reclamación presidencial ante este Tribunal Constitucional, solo cabe entender que la aludida representación efectuada en su momento por la Contraloría General de la República quedó a firme, frustrando definitivamente el ingreso de la citada norma reglamentaria al ordenamiento jurídico en vigor."

\section{Disidencia de los Ministros Venegas Palacios y Aróstica Maldonado}

Los Ministros Marcelo Venegas e Iván Aróstica estuvieron por acoger el requerimiento de inaplicabilidad interpuesto. Luego de reiterar lo manifestado en la prevención anteriormente reseñada, identifican tres posibles órbitas de conflicto en el presente caso, respecto de la legalidad de las actuaciones de las municipalidades; en cuanto a la licitud del reglamento y la constitucionalidad de la ley, siendo solamente este último conflicto de competencia del Tribunal Constitucional:

" $\left.8^{\circ}\right)$ Que, con lógica reversa y en orden creciente de significación, cabe separar los tres problemas jurídicos entrelazados en este proceso. El primero concierne a la validez de los actos edilicios en que se ha procedido nominativamente a cobrar esta contribución de patente municipal, aplicando para ello el reglamento y la ley de que se trata. La juridicidad de tales actos administrativos individuales es discutida en el respectivo reclamo de ilegalidad regulado por la Ley $N^{\circ} 18.695$ (articulo 151), por lo que en el mismo no pude intervenir el Tribunal Constitucional.

El análisis en forma ascendente permite identificar una segunda cuestión, relativa ahora a la licitud de la definición general formulada por el articulo $2^{\circ}$, letra c), del DS $N^{\circ} 484$, de 1980, en la cual establece qué es lo que "se entenderá" por "actividades terciarias" de manera amplia y residual. Es obvio que por la vía del artículo 93, $N^{\circ}$ 6, de la Carta Fundamental, no puede esta Magistratura examinar la regularidad de dicho acto reglamentario dictado por el Presidente de la República a través del Ministerio del Interior.

Pero, restado lo anterior, aparece que sí compete a este órgano jurisdiccional pronunciarse sobre la constitucionalidad del DL $N^{\circ} 3.063$, de 1979, y en primer lugar respecto a su 
artículo 23, inciso tercero, donde prevé que "(e)l Presidente de la República reglamentará la aplicación de este artículo".

En cuanto al primero de los vicios invocados, la vulneración de la reserva legal en materia tributaria, los disidentes estiman que corresponde acoger el requerimiento, por cuanto la remisión que se hace al Presidente de la República es amplia e indefinida, lo que vulnera la Constitución:

"10 ) Que, no obstante, el desarrollo práctico del precepto examinado permite revelar su propia inconstitucionalidad, al remitirse a un reglamento de manera ambigua y abierta a la más amplia discrecionalidad fiscal, atendidos los diferentes alcances que puede atribuirse a la voz "aplicar".

Es esta norma legal, entonces, en su consustancial deficiencia, la causa que posibilita el siguiente efecto anticonstitucional: ahorrándose acotar aquellos aspectos secundarios o de detalle que demanda una "aplicación" estrictamente tal, su parca redacción facilita que un reglamento -libre de moderaciones legales-venga a "establecer" un nuevo hecho gravado, cual es que la sola circunstancia de obtener una ganancia, aunque no sea fruto del ejercicio efectivo de alguna actividad terciaria, de todas maneras está afecta al pago de contribución municipal;"

Lo anterior se corrobora en el caso concreto de autos, en donde la Municipalidad aplicó la patente municipal, basándose en la ley, pero también en la definición que se hace por parte del reglamento, lo que deja en claro que es el reglamento el que termina de configurar la obligación tributaria:

"12\%) Que, si por los efectos se llega a las causas, es revelador en este caso que el ordinario alcaldicio $N^{\circ} 10 / 215$ sea resultado de aplicar las normas refutadas, a cuyo amparo se concluye que las sociedades de inversión pasiva se encuentran gravadas con contribución municipal, según revela el razonamiento de fs. 37 a 49 de estos autos (informe municipal a la Corte de Apelaciones de Santiago).

Allí puede verse que, para arribar a dicha conclusión, la Municipalidad de Las Condes ha tenido que basarse en el citado artículo 23 de la Ley de Rentas Municipales y -necesariamente- en la aludida "definición" amplia formulada en el artículo $2^{\circ}$, letra c), del reglamento aprobado por DS $\mathrm{N}^{\circ} 484$, de 1980, por entender que en estas dos normas mancomunadas se encuentra la causa que explica y respalda su proceder;"

Por último, hacen los Ministros disidentes una referencia a la disposición sexta transitoria de la Constitución, y su relación con las normas que establecían tributos especiales o los regulasen, sin que tuviesen rango de ley:

"22) Que, finalmente y aunque no sea un asunto invocado por los requirentes, conviene recordar que es un principio no controvertido del derecho público que las normas de excepción, como lo son las disposiciones transitorias de la Constitución, deben aplicarse rigurosamente a los casos en ellas previstos y no a otros, sea por analogía o extensión, conforme al principio de la interpretación restrictiva de los preceptos de excepción, unánimemente aceptado por la doctrina y aplicado invariablemente por este Tribunal; 
23) Que la Disposición Sexta Transitoria de la Carta Fundamental señala que, no obstante lo dispuesto en el inciso tercero del $N^{\circ} 20$ del artículo 19, "mantendrán su vigencia las disposiciones legales que hayan establecido tributos de afectación a un destino determinado, mientras no sean expresamente derogadas";

$2^{\circ} 4^{\circ}$ Que, por la claridad del texto constitucional, es obvio que la señalada excepción cubre unicamente a las normas legales y no a las reglamentarias o de inferior jerarquía, que a la entrada en vigor de la Constitución establecían tributos afectados a un destino determinado;

25\%) Que, en consecuencia, toda otra norma concerniente a tributos afectados a un destino determinado existente a la fecha de entrada en vigor de la Carta Fundamental, que por su jerarquía o por su contenido entró en contradicción con lo establecido en la nueva Constitución, no pudo subsistir con posterioridad al 11 de marzo de 1981."

\section{Conclusión}

La cuestión de los obligados al pago de patente municipal ha sido objeto de diversa jurisprudencia judicial y administrativa, que no ha estado exenta de variaciones en el tiempo. La Contraloría General de la República había sostenido que las sociedades de inversión solamente estaban obligadas al pago en el caso de que efectivamente se realizaran actividades secundarias o terciarias, excluyendo por tanto a las denominadas sociedades de inversión pasiva. Sin embargo, en dictamen de 16 de noviembre de 2012, el órgano contralor reconsideró su doctrina, señalando:

"En este contexto, para determinar si una sociedad de inversión de aquellas a que se refieren los peticionarios, está sujeta al pago de la referida patente el municipio-mediante sus procesos de fiscalización, los documentos que les sean acompañados por el contribuyente y la información que les proporcione el Servicio de Impuestos Internos- debe precisar si las actividades previstas en su objeto social están comprendidas dentro del hecho gravado definido en la ley, teniendo en consideración que si el objeto social incorpora, contiene, describe o permite la realización de actividades lucrativas, estas configuran hechos gravados de acuerdo con lo previsto en el artículo 23 de la Ley de Rentas Municipales."

Esta decisión de la Contraloría General de la República, recoge la jurisprudencia reiterada de la Corte Suprema, que en varias ocasiones dejaba sin efecto dictámenes del órgano contralor. Así, por ejemplo, en sentencia de causa Rol No 5984-2012, de 6 de noviembre de 2012, tomada en consideración por el órgano contralor, la Corte Suprema señalaba:

"que para determinar si una sociedad es sujeto pasivo del pago de patente municipal es indispensable precisar si la actividad que ésta realiza está comprendida dentro del hecho gravado definido en la ley en atención al objeto social de la sociedad que se trate. Por lo tanto, es claro que si el objeto social incorpora, contiene, describe o permite la realización de actividades lucrativas, configuran éstas hechos gravados de acuerdo con lo previsto en el artículo 23 de la Ley de Rentas Municipales.” 
FRANCISCO ZÚÑIGA URBINA - Revista de Derecho Público - Vol. 79, $2^{\circ}$ Sem. 2013, pp. 239-248

Fallo que incluyó una exhortación directa a la Contraloría General de la República, en orden a que debía adecuar su doctrina a lo resuelto por la Corte Suprema. 